\title{
Solubilities of Salen Derivatives and Their Cobalt Complex in Liquid and Supercritical $\mathrm{CO}_{2}$
}

\author{
Seunghyun Koh, Byungwan Jeon, Hakwon Kim, Kwangheon Park, and Hongdoo Kim" \\ Green Nuclear Research Lab. EIRC., Kunng /lee University. Yongin, Kyungkido t+19-701. Korea \\ Recened Januay 20,2003
}

\begin{abstract}
The solubility of N.N'-Bis(salicylidene) ethylenediamine ( $n$-salen) and N.N'-bis(3,5-di-tert-butylsalicylidene) ethylenediamine ( $t$-butyl-salen) was studied with in-sint UV-VIS spectrometer. $n$-Salen is $3-5$ times more soluble than $t$-butyl-salen in liquid or supercritical $\mathrm{CO}_{2}$. This behavior may be attributed to Lewis acid-base interaction between salen and $\mathrm{CO}_{2}$. The chelation of salen with $\mathrm{Co}^{-1}$ ion in supercritical condition was confirmed to be fast enough above room temperature. However, the metal ion extraction capability of $t$-butyl salen is relatively poor because of its low solubility and ionic nature of complex.
\end{abstract}

Key Words : Solubility. Salen, Supercritical $\mathrm{CO}_{2}$, Fiber optics

\section{Introduction}

The potential usages of carbon dioxide $\left(\mathrm{CO}_{2}\right)$ as an environmentally benign solvent have been realized on many commercial applications ${ }^{1-3}$ such as food process, cleaning protocol in microelectronics and dry-cleaning industries, nuclear waste extraction. Supercritical $\mathrm{CO}_{2}\left(\mathrm{sc}-\mathrm{CO}_{2}\right)$ modified by the addition of the complexing agent has been utilized in the extraction of metal ions from various solid and liquid matrices. ${ }^{4,5}$ In order to be successful supercritical fluid extraction (SFE) of metal ions, several important requirements exist such as high formation constant of metal complexes. fast kinetics, and good solubility and stability of both the chelating agent and the metal chelate in supercritical fluid. Various chelating agents, such as dithiocarbamates, $\beta$-diketones, crown ethers, and organophosphorous compounds have been used for extracting metal species in supercritical $\mathrm{CO}_{2}{ }^{6,7}$ Nevertheless, many chelating agents have drawbacks and problems relating to metal ion extraction in $\mathrm{CO}_{2}, i . e$, poor stability and solubility, and material cost. In order to overcome these problems, new effective chelating agent must be designed and selected. For this purpose, N.N'bis(salicylidene) ethylenediamine( $n$-salen) has been suggested as a new chelating agent of metal ions in $\mathrm{sc}-\mathrm{CO}_{2}$ because of easy synthesis and its well-known metal complex. ${ }^{8.9}$ In this study, solubility and reactivity of two salen derivatives and their metal complex in liquid or supercritical $\mathrm{CO}_{2}$ were investigated for the extraction of metal ions.

\section{Experimental Section}

Materials. All chemicals were purchased from Aldrich and used as received except salen derivatives. Comınercial grade and $99.99 \% \mathrm{CO}_{2}$ gases were used for $\mathrm{CO}_{2}$ experiment. N.N'-Bis(salicylidene) ethylenediamine ( $n$-salen) and $\mathrm{N}_{,} \mathrm{N}^{\prime}-$ bis(3,5-di-tert-butylsalicylidene) ethylenediamine ( $t$-butylsalen) were synthesized by the literature procedure. ${ }^{8.9}$ The procedure is sumınarized in Scheme 1. Ethylenediamine $(8.3$ mmol) was added drop-wise to 2-hydroxybenzaldehyde ( $16.6 \mathrm{mmol}$ ) in fresh distilled $12 \mathrm{~mL}$ ethanol and the solution was refluxed for 2 hours under stirring. Yellow precipitate was filtered and washed with cold methanol. The resulting $n$ salen was recrystallized in ethylacetate hexane and dried. $t$ Butyl-salen was synthesized similarly with 3,5-di-tert-butyl2-hydroxybenzaldehye. Cobalt complex ( $t$-butyl-salen-Co) was easily made in hot methanol with $\mathrm{Co}\left(\mathrm{NO}_{3}\right)_{2} \cdot 6 \mathrm{H}_{2} \mathrm{O}$ and $t-$ buty]-salen.

Apparatus and Sample Preparations. The apparatus used in this experiment was designed by Hunt et al..$^{10}$ and was summarized in Figure I. It can be operated up to 300 bar of pressure and temperature from ambient to $100^{\circ} \mathrm{C}$. The syringe pump (ISCO Model 260D. U.S.A.) was used to deliver $\mathrm{CO}_{2}$ to the reactor cell, and control and monitor the operating pressure with the accuracy of 0.1 bar. Using the external pressure gauge (Keller, Model Leo-2, Swiss), the pressure monitor of the syringe pump and pressure transducer were recalibrated. Jo control the reactor cell temper-

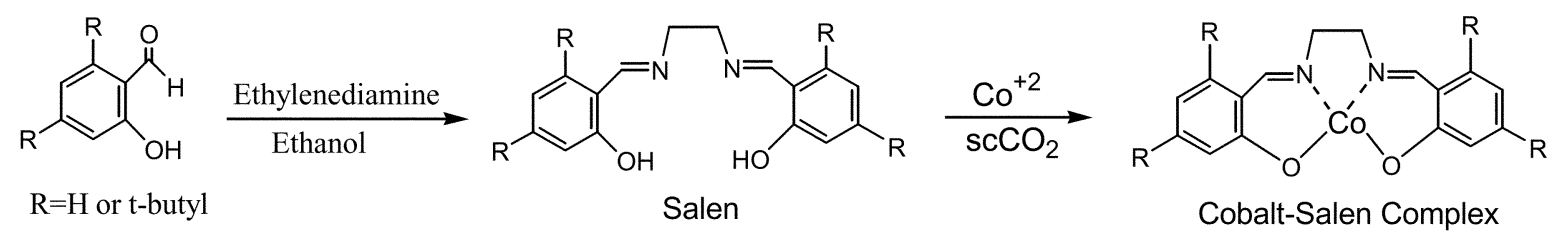

Scheme 1

"Corresponding Author, e-mail: hdkim(akhu.ac.kr 


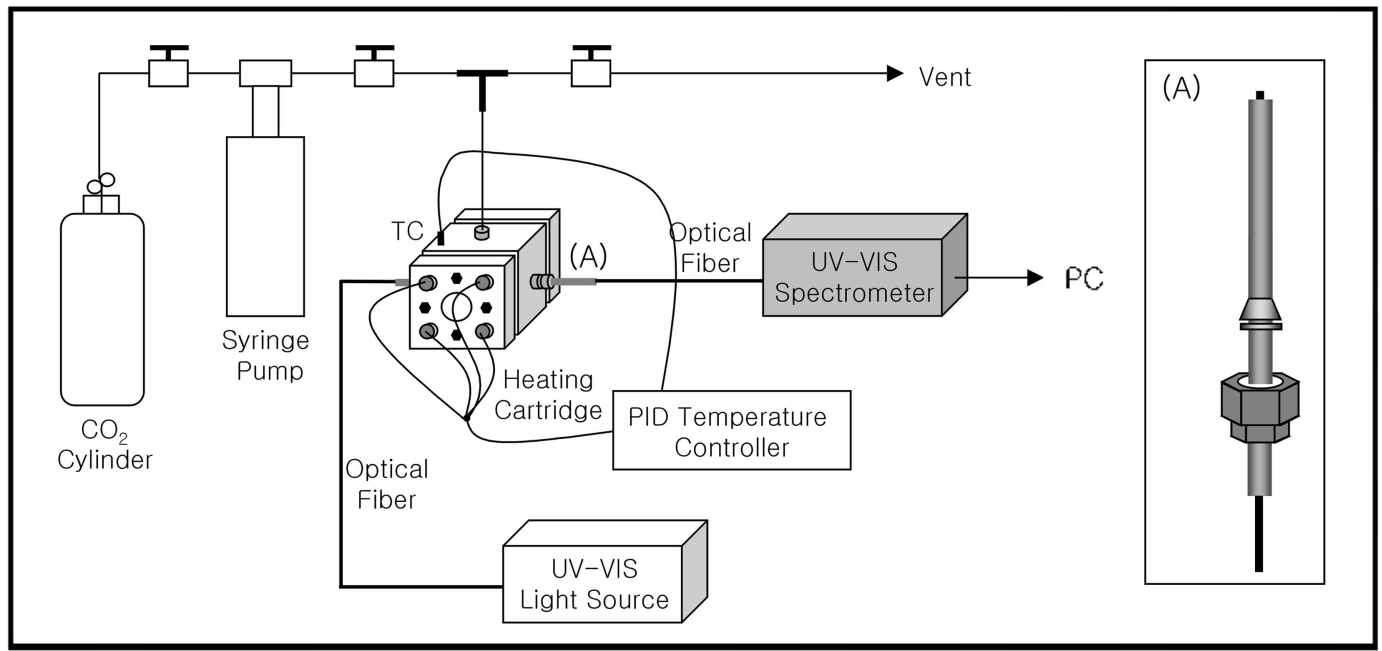

Figure I, Schematics of high pressure reactor cell with optical libers for UV-VIS measurement. Inset shown is the details of the fiber coupler with a PLEK tubing and fittings.

ature, home-build temperature controller was used. With 4 beating cartridges (Omega, U.S.A.), thermocouple and PID controller (Hanyang, Korea), the reactor cell temperature can be controlled and maintained within $0.1^{\circ} \mathrm{C}$ accuracy. The 58 $\times 58 \times 58 \mathrm{~mm}$ vessel block with $15 \mathrm{~mm}$ diameter hollow cylinder was machined from type 316 stainless steel. To achieve the sealing of the view ports. Teflon gaskets were lined on the body of the reaction vessel and $7 \mathrm{~mm}$ sapphire window at each end was bolted down to the body of reaction vessel using another piece of stainless steel block with a 15 mom diameter opening. Two 1/16 inch holes to the middle of cell were precisely drilled in an aligned manner at the right angle of the hollow cylinder and were machined to accept 1 i 16 inch male connectors in order to fit optical fibers as shown in Figure 1(A). One end of the each fiber with SMA906 connector connected to the CCD array UV-VIS spectrometer (Avantest AVS-USB2000) or UV-VIS source (Avantest DH-2000) and the other end was inserted into the cell through $1 / 16$ inch J'EEK tubing and ferrule fitting as shown in Figure $1(\mathrm{~A})$. Once the ferrule was set at the particular length from the end of fiber, the optical pathlength between the pair of fibers was fixed. Since the concentration range of the materials under study may be very large, several optical fiber pairs with different pathlengths were prepared. In this experiment, 5 different optical fibers were prepared so that the path lengths between the inserted fibers could be varied from $1 \mathrm{~mm}$ to $15 \mathrm{~mm}$. UVVIS spectra were obtained by a given path-length optical fiber pair inserted into the cell and the path-length was calibrated with known concentrations. 4 different n-salen solutions of known concentrations in hexane were prepared to calibrate the path-length between the mounted fibers. The comparison between the measured UV absorbance of the solutions with regular UV-VIS spectrometer and fiber-optics UV-VIS spectrometer gave the path-length between optical fibers. From the absorbance vs. concentration plot of solutions, the ratio of the slopes between two sets of measurements gave the path-length of a given fiber pairs. With this method, the accuracy of path-length was confirmed to be better than $5 \%$. Due to the fiber-optics cut-off below $250 \mathrm{~nm}$, however, peaks below the limit cannot be observed. With this type of reactor cell, in-situ UV-VIS spectrum and cell reaction process can be easily monitored. The amount of metal ions after extraction was analyzed by ICP method (LГE:MAN ABS Inc, Model DR : SPГC).

To test the solubility, the known concentration solution of salen was prepared in methanol and excess amount of the solution was added to the vessel and the solvent was evaporated. Upon adding the $\mathrm{CO}_{2}$, the concentration and the solubility of a given salen was calculated based on the absorbance of UV-VIS spectra. In order to obtain the equilibrium solubility at given pressure and temperature, in-situ absorbance of salen in $\mathrm{CO}_{2}$ fluid was monitored until no further change in the absorbance occurred. Its equilibrium absorbance was measured at another $30 \mathrm{~min}$ later and its equilibrium solubility was determined. For cobalt ion extraction, $1 \mathrm{~cm} \times 1 \mathrm{~cm}$ filter paper (Whatmann No. 2) was used as a substrate. With $1000 \mathrm{ppm} \mathrm{Co}^{++}$ion standard solution, $1.60 \mathrm{ppm}$ sample specimen for $\mathrm{Co}^{++}$ion extraction was prepared. After $\mathrm{Co}^{--}$ion extraction experiment, $\mathrm{ICP}$ analysis was followed after the acid digestion with $\mathrm{HNO}_{3}$ and the reported extraction efficiency was calculated from the $\mathrm{ICl}^{2}$ result.

\section{Results and Discussions}

Figures 2 and 3 show in-situ UV-VIS spectra of $n$-salen and $t$-butyl-salen, respectively. Both salen derivatives dissolve gradually up to a few $\mathrm{mM}$ order as $\mathrm{CO}_{2}$ pressure increases. Normally, salen derivatives have another peak below $270 \mathrm{~nm}$ which is not shown in Figures 2 and 3 due to the fiber-optics cut-off. The peak between $280 \mathrm{~nm}$ and $380 \mathrm{~nm}$ was used to calculate the concentration of salen in either liquid or supercritical $\mathrm{CO}_{2}$. The solubility of two salen derivatives at $21^{\circ} \mathrm{C}$ and $61^{\circ} \mathrm{C}$ are summarized in Figure 4 . In liquid $\mathrm{CO}_{2}$ at

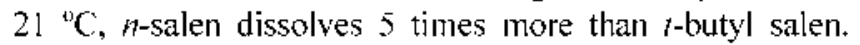




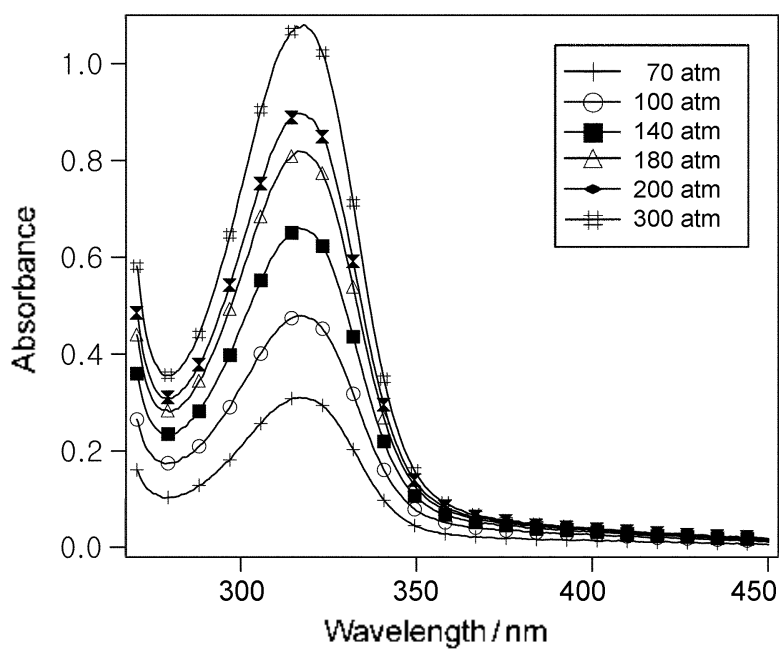

Figure 2, LIV-VIS spectra of $n$-salen as finction of $\mathrm{CO}$ - pressure at $21^{\circ} \mathrm{C}$.

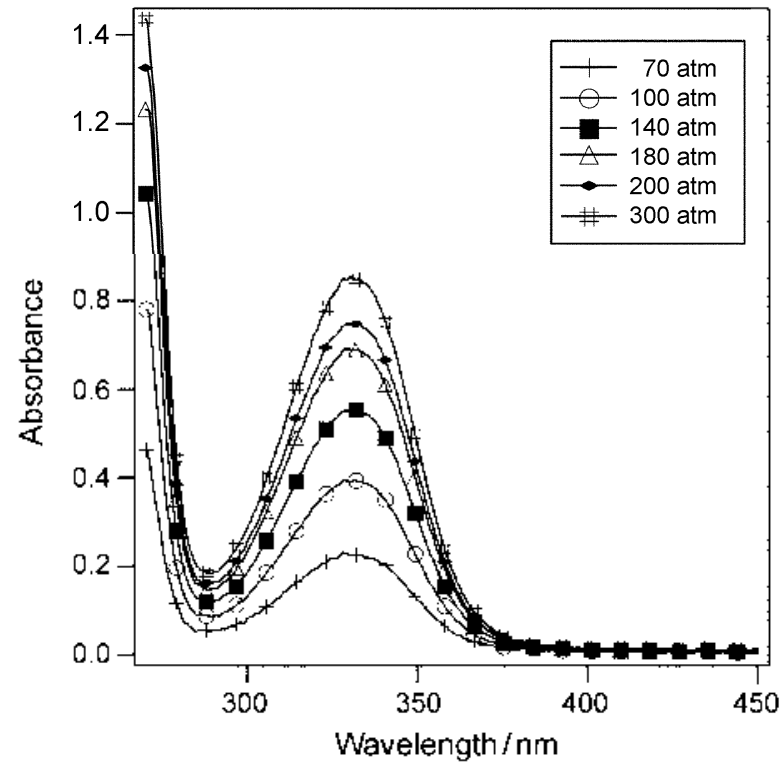

Figure 3. UV-VIS spectra of $t$-buty l-salen as function of $\mathrm{CO}_{2}$ pressure at $21^{\circ} \mathrm{C}$.

Considering $n$-salen is sparingly soluble in hexane whereas $t$-butyl salen dissolves rather well, it is rather surprising that the solubility of $n$-salen in $\mathrm{CO}_{2}$ solvent is better than that of $t$-butyl salen. Perhaps, this may be due to Lewis acid-base interaction between salen and $\mathrm{CO}_{2}$. However, in the case of $t$-butyl-salen, because its four $t$-butyl groups may prevent the acid-base interaction, its solubility is less compared to $n$ salen. At $61^{\circ} \mathrm{C}$, $t$-butyl salen rarely dissolves below 100 bar and from 180 bar its solubility surpasses that of liquid state mainly due to the density difference as pressure increases.

Complex formation of salen derivatives were reported in many literatures ${ }^{9.11 .13}$ and it was confirmed using methanol solution of salen derivatives and $\mathrm{Co}\left(\mathrm{NO}_{3}\right)_{2}$ in this laboratory. However, the solubility of salen-complex derivatives in liquid and supercritical $\mathrm{CO}_{2}$ is little known and recently it was reported that $n$-salen complex is not soluble in sc-

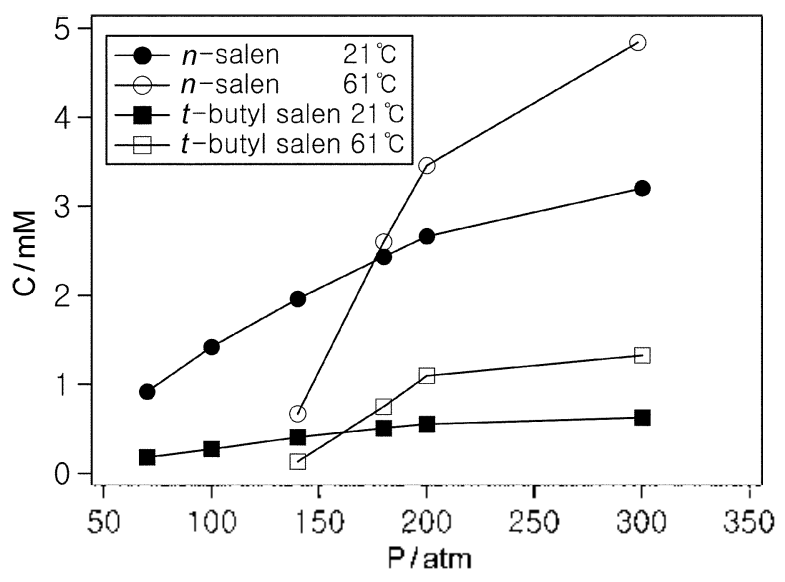

Figure 4. The solubility of $n$-salen and $t$-butyl-salen al various pressures.

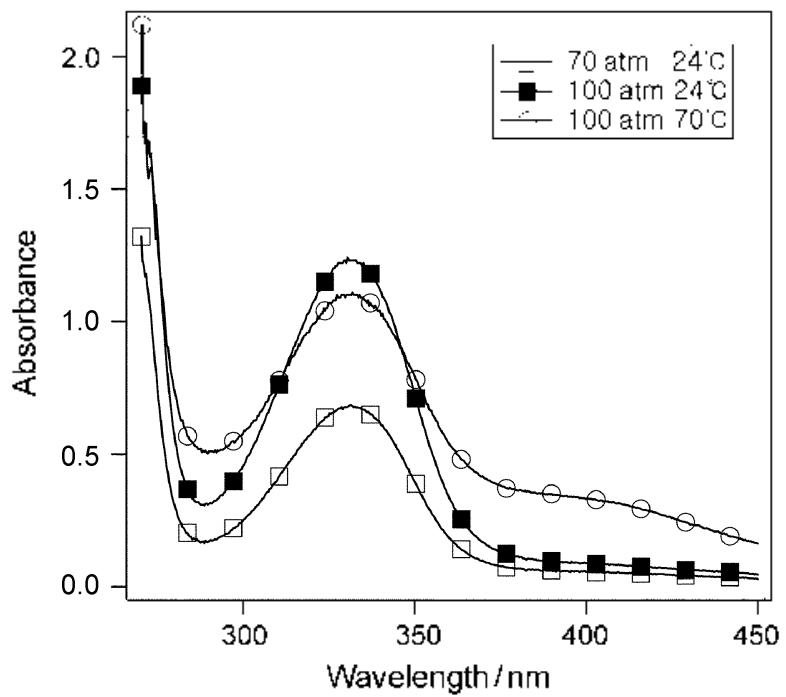

Figure 5. t-Butyl salen-Co' complex formation with the concentration ratio of $\mathrm{Co}^{++} / \mathrm{salen} 1$.

$\mathrm{CO}_{2}{ }^{1.4}$ [n order to test the complex formation of $t$-butyl salen with $\mathrm{Co}^{+-}$ion, the sample was prepared as follows: the known amount of $\mathrm{Co}\left(\mathrm{NO}_{3}\right)_{2}$ in methanol was added to the reaction vessel and the solvent was evaporated. Also, the calculated amount of solid $t$-butyl salen was added to the reaction vessel. Since the purpose of this experiment is to confirm the complex formation using in-sitn UV-VIS, the possible $\mathrm{Co}\left(\mathrm{NO}_{3}\right)_{2}$ loss during heating or residual methanol were not checked.

As shown in Figure $5, \mathrm{CO}_{2}$ fluid was added into the cell and the absorbance peak of $t$-butyl salen at $330 \mathrm{~nm}$ appeared at 70 bar. At $24^{\circ} \mathrm{C}$. no complex formation with $\mathrm{Co}^{++}$ion was observed even at the pressure of 100 bar indicating that the complex formation kinetics is slow at this temperature. At 70 "C, however, new peak around $400 \mathrm{~nm}$ was confirmed to be originated from $t$-butyl-salen-Co complex. When an excess amount of $\mathrm{Co}^{-+}$ion was used, clean $t$-butyl-salen-Co peak around $390 \mathrm{~nm}$ is observed as shown in Figure 5 and free $t$ buty]-salen peak at $330 \mathrm{~nm}$ completely disappeared because of the its complexation with $\mathrm{Co}^{-+}$ion. 


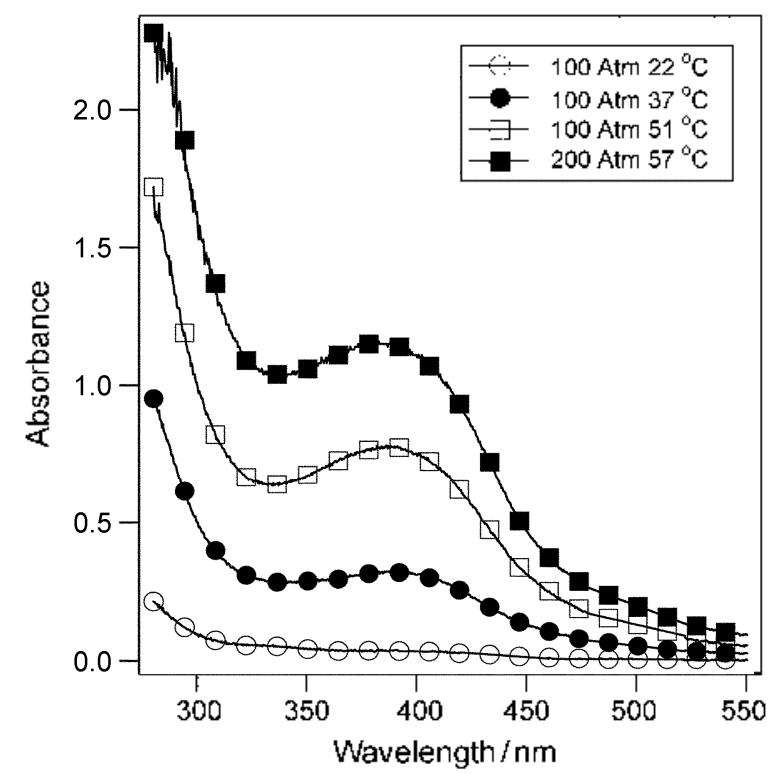

Figure 6. $t$-Buty] salin- $\mathrm{Co}^{-1}$ complex formation with the concentration ration of $\left(\mathrm{Co}^{+-} / \mathrm{sal}\right.$ en 20 . No liree $f-$ butyl-salen peak at $330 \mathrm{~mm}$ is observed.

Since $t$-butyl salen can form a complex with cobalt ion in supereritical $\mathrm{CO}_{2}$, it may be used as an extracting material for the supercritical $\mathrm{CO}_{2}$ metal extraction. With a filter paper containing $1.60 \mathrm{ppm} \mathrm{Co}^{-+}$ion, the extraction ability of $t^{-}$ butyl salen was tested. Without any modifier such as water or methanol to increase the extraction ability of salen, no cobalt ion extraction was observed, indicating $t$-butyl-salen alone does not have an ion solvating power or/and the substrate have a strong adhesion force toward metal ion. In order to increase solvating power of $\mathrm{CO}_{2}$ toward metal ions, methanol has been used as solvent moditier and is very soluble with $\mathrm{CO}_{2}$ fluid. In many applications, in fact, $5 \%$ methanol added $\mathrm{CO}_{2}$ has commonly been used. As shown in Figure 6 , with 20 or $200 \mu \mathrm{l}$. methanol damped specimens, $\mathrm{Co}^{+-}$ion was statically extracted from the filter paper substrate for 1 hour. Since methanol modified $\mathrm{CO}_{2}$ can extract the metal ion, the extraction efficiency of methanol itself was checked with the same experimental condition without any salen. It was found to be about $40 \%$ extraction efficiency with $200 \mu \mathrm{L}$ methanol modified $\mathrm{CO}_{2}$ fluid. All efficiency shown in Figure 7 with $t$-butyl-salen $/ 200 \mathrm{ml}$ methanol modified $\mathrm{CO}_{2}$ fluid is above $60 \%$ but it is not quite a promising result. However, this result is understandable. The solubility of $t$-butyl salen itself is about order of $1 \mathrm{mM}$ in either liquid or supercritical $\mathrm{CO}_{2}$. Once it is chelated with $\mathrm{Co}^{+-}$ion, the solubility of $t$-butyl salen $-\mathrm{Co}^{+-}$complex will decrease substantially because of its ionic nature. To overcome this problem, more $\mathrm{CO}_{2}$-phillic group of salen moiety and the $\mathrm{CO}_{2}$-soluble compensating counter ion must be introduced. Further studies of salen derivatives with $\mathrm{CO}_{2}$ phillic group will be reported.

\section{Conclusion}

The solubility of $n$-salen and t-butyl-salen in liquid

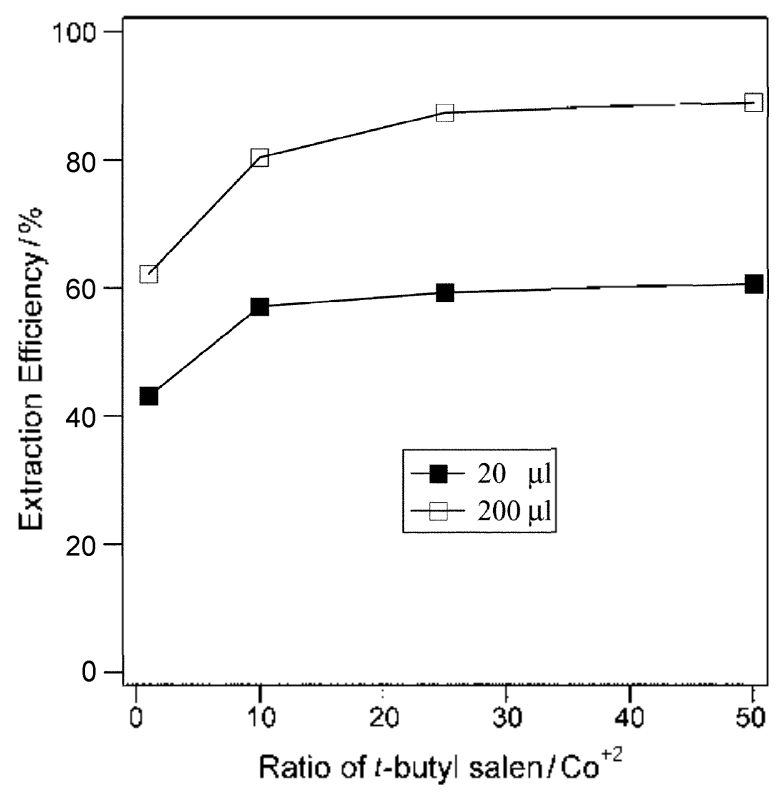

Figure 7. $\mathrm{Co}^{++}$ion extraction efficioney of $t$-butyl-salen $w$ ith 20 and $200 \mu \mathrm{l}$, methanol damped specimens.

supercritical $\mathrm{CO}_{2}$ was studied with in-situ UV-VIS spectrometer. Unexpectedly, $n$-salen is 3-5 times more soluble than $t$-butyl-salen in liquid or supercritical $\mathrm{CO}_{2}$. This behavior may be attributed to l ewis acid-base interaction between salen and $\mathrm{CO}_{2}$ and bulky $t$-butyl groups in $t$-butyl-salen hinder the acid-base interaction so that its solubility is less. The chelation of salen with $\mathrm{Co}^{+-}$ion in supercritical condition was confirmed. However, the metal ion extraction capability of $t$-butyl-salen is poor because of its low solubility and ionic nature when the chelation occurs

Acknowledgement. This work is supported by National Research Laboratory Fund, MOST and also supported by EIRC program, MOCIE of Korea.

\section{References}

1. Eckett. C. A.: Knutsons. B. I.: Debenedetli. I'. G. Aanme 1996. 373. 313.

2. Wells. S, 1.: DeSimmont, J, . A. Angew. (hem. Int. Ed. 2001, 4f) 518.

3. Mesieuno. A. J.: Beckmaus. J:. J.: Rusell. A. J. Chem. Rev 1999. 99. 623

4. Laintz. K. E.: Yu. J. I.: Wai. C. M. Cart Anal. Chem 1992. 64 311.

5. Wang I.: Marshall, W. D. Anal. ('hem. 1994, 66, 3900.

6. Wai. C. W.: Wang. S. Yu, I.-I. And. Chem. 1996.68. 3516.

7. Wai. C. M.: Waller. 13. Ind. Eng. Chem. Res. 2000. 30. 4837.

8. van Dort. H. .: Guerson. H. J. Recl. Tran: (him. Pans-Bats 1967. 86.520 .

9. Chang. S.: Heid. R. M.: Jacobson. E. N. Tetrohedron Lett. 1994. 35,669 .

10. Ilunt. Г.: Ohda 11.: Wai. C. . 1. Kev. Sci. Instrum. 1999, 70. 4661.

11. Pietikainem. P.: I latikarainen. A. J. Wh. Cakd. A 2002. /80. 59.

12. Liang. S.: Bu. X. R.J. Org. ('hem. 2002.67. 2702.

13. Jacobson. E. N. In Catolvic Asymutwic Swmthesis: Ojima. L.. Ed.: VCH: Nisw: York. 1993: Chapter 4.

14. Music, (i. T.: Wei. M.: Subrameniem, B.: Busch. D. II. Inorg. (hem. 2001, 40,3336 . 